\title{
PROCESO DE INTEGRACIÓN DE AMÉRICA LATINA Y EL CARIBE
}

\section{PROCESS OF INTEGRATION OF LATIN AMERICA AND THE CARIBBEAN}

\section{Luis Carlos Herrera M.}

\section{RESUMEN}

El presente artículo presenta un análisis del proceso de integración de América Latina y el Caribe, su nivel de complejidad, los distintos mecanismos existentes y su importancia para el desarrollo de los países de la región y el bienestar de la población. Para hacerle frente a este desafió, se recurrió en primera medida al análisis histórico (heurístico, crítico y síntesis) de los procesos de integración a nivel de Norte América, Centroamérica, Sur América y el Caribe. La segunda decisión metodológica, implicó estudiar desde la sociología política, la experiencia propia de cada mecanismo de integración; identificando logros, dificultades, retos y perspectivas, frente a la composición geopolítica de la región. Los resultados apuntan a que el proceso de integración latinoamericana y caribeña ha tomado décadas; ha sido un largo proceso con avances y retrocesos. En el proceso de integración se han tenido que superar situaciones difíciles, que provocaron la incorporación de temas como: la desigualdad social, el cambio climático, la seguridad alimentaria, justicia, democracia, participación ciudadana, corrupción.

PALABRAS CLAVE: AMÉRICA LATINA * CARIBE * INTEGRACIÓN REGIONAL * SOCIOLOGÍA POLÍTICA* POLÍTICA DE DESARROLLO

\section{ABSTRACT}

This article presents an analysis of the integration process of Latin America and the Caribbean, its level of complexity, the different existing mechanisms and their importance for the development of the countries of the region and the welfare of the population. In order to face this challenge, the historical analysis (heuristic, critical and synthesis) of integration processes at the level of North America, Central America, South America and the Caribbean was used first. The second methodological decision involved studying from political sociology, the experience of each integration mechanism; identifying achievements, difficulties, challenges and perspectives, as opposed to the geopolitical composition of the region. The results suggest that the process of Latin American and Caribbean integration has taken decades; It has been a long process with advances and

Universidad Santa María La Antigua (USMA) y Sistema Nacional de Investigación (SNI) de la Secretaria Nacional de Ciencia y Tecnología (SENACYT), ambos de Panamá.

lherreram@usma.com.pa 
setbacks. In the process of integration have had to overcome difficult situations, which led to the incorporation of topics such as: social inequality, climate change, food security, justice, democracy, citizen participation, corruption.

KEYWORDS: LATIN AMERICA * CARIBBEAN * REGIONAL INTEGRATION * POLITICAL SOCIOLOGY * DEVELOPMENT POLICY

\section{EL CAMINO RECORRIDO EN EL PROCESO DE INTEGRACIÓN}

Las ideas integracionistas surgen en la época poscolonial, a finales del siglo xviII $y$ durante el xIx, para impulsar el comercio y la economía; las naciones recién independizadas buscaron crear su propia identidad, sobre la base de consolidar los lazos históricos, políticos y culturales. El despertar de la consciencia latinoamericanista, coincidió de este modo, con el descubrimiento de las consecuencias que acarreaban para los países de la región, las nuevas formas de penetración económica y política de las potencias capitalistas en su etapa imperialista (Fernández, 2014).

La visión de una Patria Grande del Libertador Bolívar, sustentada en el Manifiesto de Cartagena de 1812 y en la Carta de Jamaica del 6 de setiembre de 1815, exponía sus ideas de una sola y gran nación, conscientes que se está hermanado no solo por nuestras raíces culturales, religiosas y un idioma; reconociendo que tenemos diferencias que nos separan, por lo cual se debe lograr un solo gobierno (Juventud, 2009).

En el Congreso Anfictiónico de Panamá del 22 de junio de 1826, después de liberar a Bolivia, último bastión español, junto al mariscal Antonio José de Sucre; el Libertador plantea la defensa de la soberanía de todas las naciones y la creación de estructuras y ciudadanía americana. En Cuba, José Martí defiende estas ideas bolivarianas, y en Centroamérica, las luchas del pueblo nicaragüense durante el siglo xx, bajo el liderazgo de Augusto Cesar Sandino, llama a realizar el sueño del libertador Simón Bolívar el 20 de marzo de 1929 (García, 1979).

En 1959, en Montevideo, el líder de la Revolución Cubana manifestó en un fragmento de motivadora intervención:
Unámonos primero en pos de nuestros anhelos económicos, en pos del mercado común y después podremos ir superando las barreras aduaneras, y algún día las barreras artificiales habrán desaparecido. Que en un futuro no muy lejano, nuestros pueblos puedan a abrazarse en una América Latina unida y fuerte. Ello será un gran paso de avance hacia la unión política futura (Castro, 1959) ${ }^{1}$.

Para Fidel Castro, la integración debe responder a un proceso realista, consciente $y$ con temas puntuales que permitan alcanzar esa unidad política en América Latina y el Caribe, con destino y características propias, donde existen más elementos que nos unen que los que nos separan.

A pesar de las disparidades que pueden presentarse de una subregión a otra, en sus economías y problemas sociales; esto no ha sido un impedimento para proponer sistemas de integración, unos con mayores resultados que otros, pero todos bajo la espada de la falta voluntad política real para que los mismos respondan a los intereses de las poblaciones.

En los distintos momentos en la evolución del proceso de integración, los organismos creados (un ejemplo de ello en Centroamérica, es el PARLACEN) muestran avances, retrocesos $y$ crisis; desde los años 60, se producen acontecimientos para lograr su institucionalización que se mantiene en la década de los 80 , con distintos contextos históricos y cuya complejidad

1 Discurso pronunciado por el comandante Fidel Castro Ruiz, primer ministro del gobierno revolucionario, en la explanada municipal de Montevideo, Uruguay, el 5 de mayo de 1959. Recuperado del sitio web http://www.cuba.cu/ gobierno/discursos/1959/esp/f050559e.html 
aborda desde intereses económicos, comerciales, culturales, hasta preservar la paz, la democracia o generar un cuerpo jurídico que atienda problemas comunes.

Las ideas expansionistas de los Estados Unidos, expresadas en la teoría del Destino Manifiesto, han sido un factor limitante para el fortalecimiento de los procesos de integración, al anteponer sus intereses, procurando debilitar la posibilidad que América Latina y el Caribe, se constituyan en un bloque de poder único de negociaciones, frente a los países desarrollados, en el marco del comercio internacional y los temas propios de la región (Fernández, 2000).

La integración debe convertirse en un espacio privilegiado para el diálogo y acuerdos políticos, económicos, sociales, culturales y ambientales; así como para la defensa de la democracia; ser una caja de resonancia de las necesidades de las personas, en donde se adopten estrategias coherentes y sostenibles, que concreten los esfuerzos tanto comerciales, económicos y sociales, como de apertura de mercados, zonas de libre comercio, uniones aduaneras, eliminación de restricciones arancelarias, políticas fiscales, monetarias, acuerdos sociales, culturales, marcos normativos y mecanismos de coordinación suprarregional.

\section{1) EVOLUCIÓN DE LA INTEGRACIÓN A NIVEL INTERAMERICANO}

América Latina, bajo el pensamiento bolivariano a procurado mantener una identidad y consolidar la integración que le permita en mejores condiciones enfrentar a las grandes potencias económicas y políticas que han mantenido la hegemonía en la región. Desde 1889 se promueve la Primera Conferencia Panamericana, como espacio de relaciones de carácter gubernamental suprarregional en la que se tiene el control ideológico y político; que evoluciona a lo que hoy es la Organización de Estados Americanos (OEA).

Durante los años 50, el desarrollo de las industrias nacionales requiere de nuevos mercados, se transforma la dinámica comercial con las alianzas entre los países del continente, de manera que en los años 60 y 70, la política exterior permite alcanzar un mayor desarrollo económico a lo interno de los países.

El auge del pensamiento bolivariano $y$ las aspiraciones de autonomía de los Estados Latinoamericanos y Caribeños, da surgimiento a los primeros sistemas integracionistas como la Asociación Latinoamericana de Libre Comercio (ALALC), El Mercado Común Centroamericano (MCCA), El Pacto Andino y La Comunidad del Caribe (CARICOM); para continuar en su avance en aspectos económicos, comerciales $y$ de los mercados.

En los años 80 y 90, se avanza con la creación del Mercado Común del Sur (MERCosuR), la Asociación de Estados del Caribe (AEC), la Comunidad Andina de Naciones (CAN), el Sistema de Integración Centroamericana (SICA), el Mercado Común Caribeño (MCC) y y durante el siguiente periodo surgen las iniciativas del Área de Libre Comercio de las Américas (ALCA), Alternativa Bolivariana para América Latina y el Caribe (ALBA) y la Unión de Naciones Suramericanas (UNASUR).

La integración se ha enfrentado distintos escenarios y problemas: políticas neoliberales, el consenso de Washington que promueve en los Estados privatizaciones, la reducción del sector público y en las inversiones sociales, la globalización, las crisis políticas, corrupción, desigualdad social, inseguridad, pobreza y la violación de derechos humanos.

Las desigualdades sociales y las asimetrías entre los países en los distintos sistemas, ha sido uno de los elementos disonantes para que los resultados puedan impactar de la misma forma los mercados y la economía de los países. En base a esta situación, países de menor desarrollo económico exigen tener un trato especial $y$ diferenciado (De la Mora y Rodríguez, 2011).

Cada proceso integracionista deja lecciones importantes, aprendizajes $y$ avances, no obstante, la población no comprende en qué medida son necesarios y hasta qué punto impactan su calidad de vida, donde la poca información del trabajo que cada uno realiza, ha sido un factor negativo. 
ÁREA DE LIBRE COMERCIO DE LAS AMÉRICAS (ALCA)

Estados Unidos en 1991 bajo la presidencia de George Bush, propone la creación del ALCA, entre sus principales objetivos se encuentran el lograr reformas económicas, la apertura de zonas de libre comercio y políticas comerciales. Esta alianza se concreta en 1994, donde logra el respaldo de los 34 países de la región.

El ALCA reúne a naciones desarrolladas y en vías de desarrollo, respondiendo a las disposiciones multilaterales de la Organización Mundial del Comercio (омc), donde se incluyen temas como: inversión en bienes y servicios, compras del sector público y políticas de competencia; agricultura y acceso a mercados; políticas de competencia y subsidios procedimientos antidumping $y$ derechos compensatorio.

Los defensores del tratado del ALCA lo han presentado como una oportunidad para desarrollar las relaciones comerciales y establecer normas arancelarias; lo cierto es que en varios años de debate, los Estados Unidos no pudieron imponer su propuesta para restablecer está área de libre comercio, ya que para las naciones del sur, su mayor interés es fortalecer MERCOSUR.

El aLCA contempla entre sus objetivos promover la prosperidad a través de la integración económica, en temas como el acceso a mercados, inversión, servicios, compras del sector público, agricultura, subsidios, políticas de competencia, entre otros; sin embargo, las asimetrías entre países, las posiciones ideológicas y el nivel de desarrollo económico, son aspectos que deben superarse para encontrar que los intereses de cada miembro y el nivel de influencia de los países desarrollados no sea determinante en el futuro del ALCA.

La integración comercial es un mecanismo para incidir en la eliminación de la desigualdad, la pobreza y otros problemas. Una zona de libre comercio, sin considerar la brechas entre países y el intercambio desigual de amplios mercados como Estados Unidos y Canadá, bajo las condiciones existentes, es impensable, aunque la presencia de México ha sido importante en este escenario, lo que ha propiciado acuerdos bilaterales, a pesar de existir posiciones a favor $y$ en contra.

\section{EL PARLAMENTO LATINOAMERICANO (PARLATINO)}

El parlatino es un organismo regional permanente $y$ unicameral, se constituye el 7 de diciembre de 1964, en Perú, el cual se institucionaliza de forma permanente el 16 de diciembre de 1987. Su sede está ubicada en la ciudad de Panamá.

En este espacio regional están representados 23 países de América Latina y el Caribe, cuyos miembros son elegidos por sufragio popular en sus respectivos e integrados por delegaciones permanentes $y$ pluralista.

Sus estatutos recogen los principios que los rigen: fomentar el desarrollo económico y social, la integración latinoamericana, la defensa de los postulados de justicia social, libertad y la democracia; la igualdad jurídica de los Estados, la no intervención, la autodeterminación de los pueblos, la pluralidad política e ideológica, la condena al uso de la fuerza contra la independencia política y la integridad territorial de cualquier Estado, la solución pacífica y justa de las controversias internacionales, apegados a los principios del derecho internacional y la cooperación.

Es una institución democrática de carácter permanente, representativa de todas las tendencias políticas existentes en los cuerpos legislativos; encargada de promover, armonizar y canalizar el movimiento hacia la integración. Aspira a convertirse en un organismo político de la integración, que propicie el establecimiento de una normativa regional, que genere vínculos de trabajo e intercambio entre los países en distintos temas (Vacchino, 1990).

\section{LA ALIANZA DEL PACÍFICO (ADP)}

Es un espacio de integración económica y comercial, creada en 2011 a través del Acuerdo Marco de la Alianza del Pacífico. En junio de 2012, se instala de manera formal con la participación de México, Colombia, Perú y Chile. Posteriormente, se suscribe un protocolo adicional del Acuerdo Marco por los jefes de Estado y gobierno el 10 de febrero de 2014, en la Cumbre de Cartagena de Indias. El ALCA se presenta como una plataforma estratégica que ofrece múltiples ventajas en términos de 
servicios, capitales, inversiones y movimientos de personas, para el desarrollo y libre comercio. Ofrece una oportunidad de apertura comercial, puesto que alcanza más de 112 millones de personas de América Latina y el Caribe, sumado a que cuenta con 32 Estados Observadores.

Es una plataforma que ofrece múltiples ventajas a los países, que incluye servicios, capitales, inversiones y movimientos de personas, para el desarrollo y libre comercio, que llega a una población de más de 214 millones de personas (Alianza del Pacífico, 2015).

Esta Alianza se coloca muy cerca a los intereses económicos de Estados Unidos, contrario a los esfuerzos representados en ALBA o la CELAC.

En los últimos años se ha venido debatiendo la unificación de objetivos y estrategias para la Alianza del Pacifico y el MERcosur, especialmente en las Cumbres, que son espacios privilegiados para que los jefes de Estado y Gobierno, diriman las diferencia en búsqueda de acuerdos; por lo pronto, han logrado lo que se ha denominado una "agenda corta", que define objetivos del comercio, la utilización de medios tecnológicos para la certificación o aspectos migratorios de sus ciudadanos. Entre ambos representan más del $80 \%$ del comercio exterior de América Latina y algo más del 90\% del PIB regional, según datos de la CEPAL (EFE, 2015).

La duplicidad de esfuerzos debe ser revisada integralmente. El espíritu y los anhelos que crearon estos bloques, se basan en la misma idea de unificación de sus raíces, recursos y sueños para enfrentar con fortaleza los embates de una economía global, que se sustenta en una ideología deshumanizante.

\section{COMUNIDAD DE ESTADOS LATINOAMERICANOS Y} CARIBEÑOS (CELAC)

La Comunidad de Estados Latinoamericanos es una instancia de integración de carácter gubernamental que promueve procesos de coordinación y concertación política. Se constituye durante la iI Cumbre de América Latina y el Caribe en 2011 en la República Bolivariana de Venezuela, a la par de la xxi Cumbre del Grupo de Río, donde se aprueba la Declaración, el Plan de Trabajo de Caracas
2012 y el Estatuto de Procedimientos para el funcionamiento orgánico.

Este esfuerzo multilateral de integración, desde la diplomacia de Cumbres, tiene un impacto importante en la región, porque permite el intercambio político al más alto nivel, para atender agendas y concertar acuerdos de nuestra América. Está conformado de manera representativa por 33 los países latinoamericanos y caribeños; aspira a ser la voz autorizada a nivel global, para representar a nuestra región en temas importantes.

Es un organismo que promueve la integración de manera inclusiva y equitativa, comprometido en garantizar el desarrollo sostenido $y$ productivo en armonía con la naturaleza, respeto a los derechos humanos, la desigualdad, la pobreza, el cambio climático, la seguridad alimentaria, el derecho humano al agua, al trabajo decente, la salud integral, la educación de calidad, viviendas dignas, la migración, así como, atender las normas del comercio, el sistema financiero y los problemas de la corrupción. Asimismo, velar por los grupos indígenas, afrodescendientes, las mujeres, niñas, adolescentes $y$ personas adultas mayores. Un espacio que propone atender los problemas de la paz y la democracia con la participación de todos los actores sociales para alcanzar una arquitectura regional y una nueva forma de trabajar por el bien común (CELAC, 2016).

La participación de las naciones caribeñas en la III Cumbre de la CELAc, ha dejado de manifiesto su compromiso con la integración, como la única vía para mejorar la calidad de vida de la ciudadanía y promover la justicia y el desarrollo sostenible (Granma, 2015).

La CELAC cuenta con un alto nivel de tomadores de decisiones, habrá que esperar si mantiene el nivel de voluntad política que se requiere, en momentos que se debe de implementar una agenda de desarrollo al 2030, convirtiéndose en una oportunidad y gran responsabilidad para redefinir el futuro común de los pueblos en América Latina y el Caribe. La integración ha propiciado un diálogo importante en temas sociales, ambientales, culturales $y$ respeto a los derechos humanos, el cual debe trasladarse al escenario internacional para 
continuar impulsando un desarrollo económico que no esté alejado de la solución de los problemas de la población, a través de medidas concretas e integrales.

\section{ASOCIACIÓN LATINOAMERICANA DE INTEGRACIÓN (ALADI)}

Es un organismo intergubernamental, creado por el Tratado de Montevideo el 12 de agosto de 1980, con el objetivo de promover la integración y el libre comercio. Al igual que otros órganos de integración ha venido evolucionando desde los años 80 , siendo su antecedente la ALALC, que pasa a convertirse en ALADI en 1980.

El esfuerzo de ALADI está destinado a crear un área de preferencias económicas en la región, para concretar un mercado común latinoamericano, mediante tres mecanismos: la preferencia arancelaria regional que se aplica a productos originarios de los países; acuerdos de alcance regional para todos los países y los acuerdos parciales, con la participación de dos o más países del área. Estos acuerdos regionales y parciales, incorporan distintos temas, tanto comerciales, agropecuarios, aduaneras, financieras, ambientales, turismo, complementación económica; comercio agropecuario; cooperación financiera, tributaria, aduanera, sanitaria; preservación del medio ambiente; cooperación científica y tecnológica; promoción del turismo; normas técnicas.

Entre los principios de ALADI se encuentran: el pluralismo en materia económica y política, la convergencia progresiva de esquemas subregionales y acuerdos bilaterales hacia la formación de un mercado común latinoamericano, flexibilidad, tratamientos diferenciales con relación al nivel de desarrollo de los países miembros y multiplicidad en las formas de concertación de instrumentos comerciales.

Los países calificados de menor desarrollo económico (Bolivia, Ecuador y Paraguay) gozan de un sistema preferencial, a través de programas especiales de cooperación (ruedas de negocios, pre inversión, financiamiento, apoyo tecnológico) y de medidas compensatorias (ALADI, 2015).
ALADI viene a formar parte de la diversidad de órganos de integración que no han logrado concretar sus objetivos, ni las aspiraciones de la población y al final son pocos los países comprometidos, con poca voluntad política para definir una real integración, en una región de más de 600 millones de habitantes. Al igual que MERCOSUR y la CAN, comparten aspiraciones de fortalecer las relaciones económicas, comerciales y establecer un mercado común, aunque cada uno conserva su autonomía, no se supera el entramado complejo de duplicidad de esfuerzos y recursos.

\section{2) EVOLUCIÓN DE LA INTEGRACIÓN EN CENTROAMÉRICA}

\section{MERCADO COMÚN CENTROAMERICANO (MCC)}

El 13 de diciembre de 1960, con la firma del Tratado General de Integración Centroamericana (Tratado de Managua), se crea el Mercado Común Centroamericano (Mcc) con el compromiso de crear un mercado común, un arancel $y$ un libre comercio centroamericano para los productos producidos en la región. Es una de las iniciativas pioneras en Centroamérica, conformado por Costa Rica, Guatemala, El Salvador, Nicaragua y Honduras. Este proceso de integración se ha visto fortalecido por el apoyo del Banco Centroamericano de Integración Económica (BCIE).

La década de los 80, llamada la década perdida, muestra una Centroamérica con una gran cantidad de población en condiciones de pobreza y extrema pobreza, donde los programas de ajuste estructural y modernización, tuvieron un impacto nefasto, en una región que recién resolvía sus diferencias políticas y en algunos casos mantenían dictaduras.

En su más de cincuenta años de establecimiento, ha logrado modificaciones en los instrumentos legales que lo rigen y el reconocimiento de su importancia; las oportunidades para los países miembros en una relación comercial favorable, ampliación de sus mercados de exportaciones con la Unión Europea y recibir asistencia técnica por vía de la cooperación. 
Aunque el proceso de integración económica ha sido relevante para la región, es necesario ampliar la divulgación de las normas de integración comercial y las ventajas que brinda al mercado centroamericano de consumidores; de igual forma debe incorporar otros sectores económicos, mejorar la competitividad y preparar recurso humano especializado para intervenir en la consecución de mejores acuerdos.

El MCC ha superado los embates de la crisis financiera internacional y la competencia de otros bloques como el MERCOSUR, pero tiene que ser más competitivo, incorporar el uso de las tecnologías que faciliten la producción y el comercio y preparar recurso humano para que siga creciendo (Rubio, 2011).

EL SISTEMA DE LA INTEGRACIÓN CENTROAMERICANA (SICA)

El sica surge de la reestructuración de la Organización de Estados Centroamericanos (odeca). Se constituye mediante el Protocolo de Tegucigalpa durante la xi reunión de Jefes de Gobierno y de Estados Centroamericanos en 1991, en un esfuerzo por avanzar en la integración. El Protocolo es el tratado constitutivo marco más importante del proceso integracionista centroamericano, es un referente interesante por cuanto aborda no solo los temas económicos, sino que tiene una visión integral, social, política, cultural, ambiental, de género, con respeto a los derechos humanos. Sus objetivos de paz, libertad, democracia y desarrollo son principios que sustentan la vida normativa $y$ de desarrollo humano sostenible de la integración.

Este sistema de integración lo conforman 7 países, son miembros de pleno derecho: Costa Rica, El Salvador, Guatemala, Nicaragua, Panamá, Honduras y Belice; a partir del 2013 se incorpora República Dominicana. Tiene cuatro subsistemas en las áreas social, económica, política y cultural.

En el proceso de evolución del sica, hay que ponderar su capacidad de renovarse. En la Cumbre Extraordinaria realizada en el Salvador, el 20 de julio de 2010, los Jefes de Estado aprobaron los cinco grandes pilares de la integración: seguridad democrática, prevención y mitigación de los desastres naturales y de los efectos del cambio climático, integración social, integración económica y fortalecimiento de la institucionalidad regional (sICA, 2016).

El sica avanza en temas económicos, aduaneros, reglamentos normativos, pasaporte centroamericano, el establecimiento de una estrategia de seguridad democrática, política regional de pesca, en el desarrollo de la Micro y Pequeña Empresa, Salud. Sin duda que en una región marcada por las desigualdades sociales $y$ de género, tener la Política Regional de Igualdad con Equidad de Género (PRIEG), impulsada por el Consejo de Ministras de la Mujer de Centroamérica y República Dominicana (COMMCA), marca una diferencia con otros sistemas de integración (COMMCA, 2016).

Entre los problemas estructurales y culturales de la región se encuentra la persistencia de la pobreza, la exclusión y la marginalidad social de la población; frente a ello se trabaja en promover las autonomías políticas, económicas $y$ físicas de las mujeres.

El SICA muestra una evolución interesante y trabaja para consolidarse. En Guatemala en junio de 2015, los Ministros de Relaciones Exteriores debatieron la aprobación de una hoja de ruta para avanzar en los distintos temas vinculados a la unión aduanera, acuerdos comerciales con la Unión Europea, MERCosur; así como, la implementación de medidas de corto plazo para facilitar el comercio regional (ACAN EFE, 2015).

Con sus altas y bajas, es uno de los modelos de la integración que contempla una estructura donde se atienden distintos temas de interés para la región y más ampliamente los problemas sociales $y$ de derechos humanos, superando distintas crisis políticas que siempre son amenazas al sistema, como fueron los problemas limítrofes entre Costa Rica y Nicaragua sobre el río San Juan, donde la Corte Internacional de Justicia de la Haya reafirmó la soberanía de Costa Rica sobre el territorio en disputa.

\section{EL PARLAMENTO CENTROAMERICANO (PARLACEN)}

El Parlamento Centroamericano tiene su génesis en la iniciativa de Panamá de convocar en la Isla de Contadora a los cancilleres de México, Colombia, Venezuela y Panamá, para buscar una solución pacífica al conflicto 
centroamericano en 1983. La iniciativa de paz contó con el apoyo de la Unión Europea, respaldada por países de la región suramericana, lo que muestra que América se une frente a situaciones políticas difíciles, motivando la unidad latinoamericana que propició el surgimiento de este espacio de integración y que nace de los Acuerdos de Esquipulas.

Durante la toma de posesión del presidente de Guatemala para el período 1986-1990, Marco Vinicio Cerezo Arévalo, el 14 de enero de 1986, formaliza su propuesta a los países del SICA, de crear una instancia para analizar $y$ adoptar normativas para la convivencia pacífica y desarrollo de la región.

En los acuerdos de Esquipulas I, en mayo de 1986, se acuerda crear el Parlamento Centroamericano, designando una comisión técnica que prepara el Tratado Constitutivo; $y$ en Esquipulas II, en agosto de 1987, se acuerda colocar al PARLACEN, en símbolo de la libertad e independencia de la reconciliación, concretando su organización, estructura y funcionamiento. El Tratado Constitutivo del PARLACEN se firma en octubre de 1987 y cobra vigencia el 28 de octubre 1991, en Ciudad Guatemala, sede del mismo.

En su instalación participan 65 diputados centroamericanos, que representan 13 partidos políticos y cuenta con observadores de los Parlamentos Latinoamericanos, Andino y Europeo. Aunque se cuestiona la presencia de expresidentes con casos de corrupción o procesos judiciales; es la legitimidad de sus miembros, elegidos por votación popular en sus respectivos países, que determina su carácter democrático.

Se le reconoce como el órgano regional permanente de representación política y democrática del SICA, constituido por el Protocolo de Tegucigalpa y su objetivo fundamental es realizar la integración, promover la convivencia pacífica, en un sistema democrático representativa y pluralista, aunque, se reconoce como limitante que sus decisiones no tienen carácter vinculante; $y$ la población no comprende para qué sirve el tener esta instancia de integración (Primerísima, 2007).
Entre sus funciones se encuentran: proponer proyectos de tratados y convenios; propiciar la convivencia pacífica y la seguridad de Centroamérica; recomendar a los gobiernos centroamericanos las soluciones más viables $y$ efectivas en relación a los diferentes asuntos que, dentro de sus atribuciones, conozca y las demás que se le asignen (PARLACEN, 2015).

\section{LA CORTE CENTROAMERICANA DE JUSTICIA (CCJ)}

Es el órgano jurisdiccional principal $y$ permanente del SICA, su creación responde a la necesidad de atender jurídicamente los conflictos del proceso de integración centroamericana. La Corte inició sus funciones el 12 de octubre de 1994, en su sede en Nicaragua. Aunque su establecimiento antecede a la integración centroamericana, al ser uno de los primeros órganos del derecho internacional de derechos humanos, sus orígenes datan de principios de siglo.

Entre sus funciones se encuentran: velar por el cumplimiento de lo establecido en el Protocolo de Tegucigalpa (PT), sus instrumentos complementarios y acuerdos. Los países miembros del SICA y los distintos órganos que lo integran, deben someterse a su jurisdicción, competencia y doctrina, que tienen carácter obligatorio. Son además, responsables de dar interpretación del Derecho de Integración, tiene atribución como Tribunal Internacional en el derecho comunitario, arbitraje y órgano de consulta (ODECA, 1991).

La competencia de los temas de derechos humanos se adscriben a la Corte Interamericana de Derechos Humanos, que no excluye de su cumplimiento a los Estados, según el Protocolo de Tegucigalpa.

En materia jurídica, el SICA ha formado un Derecho Comunitario Centroamericano, con una amplia estructura jurídica, fuentes, órganos y procedimientos; que muestra su capacidad de lograr planes, acuerdos y políticas regionales en distintos temas, sinergias con otros organismos. Centroamérica se presenta en los escenarios internacionales como siCA, forjando una sola voz subregional que ha permitido visibilizar los problemas e incorporar sus demandas en las agendas. 
La Corte Centroamericana de Justicia, aporta doctrina al derecho comunitario, aunque el SICA está sometido al derecho internacional tiene sus propias formas jurídicas que adquieren carácter obligante, entre ellos: reglamentos, ordenanza, resoluciones, acuerdos, recomendaciones, directriz, declaración y opinión consultiva, $y$ un derecho complementario.

\section{3) EVOLUCIÓN DE LA INTEGRACIÓN EN SURAMÉRICA}

La integración en Suramérica ha sido diferente en su proceso de evolución a las de Centroamérica y el Caribe; es un escenario distinto entre grandes y pequeñas economías, con avances en acuerdos binacionales en distintos temas como: la explotación de la minería y energía e iniciativa para la infraestructura regional sudamericana. Es una región con grandes recursos naturales, donde cualquier desarrollo debe realizar diálogos y lograr una concertación social para garantizar los derechos ambientales y de los pueblos originarios, donde mayormente se encuentran estos recursos.

Con la elección de nuevos mandatarios $y$ juzgamientos de otros, como son los casos de Argentina, Uruguay y Brasil, se avecina una nueva etapa en las relaciones. América Latina es un territorio atractivo por su potencial económico, comercial y político para las potencias:

El territorio suramericano alcanza los 17.658.000 kilómetros cuadrados de superficie contigua, y ocupa el $45 \%$ del continente americano; en cuanto a su población hay aproximadamente 361.000.000 habitantes y su PIB alcanza anualmente aproximadamente 973.613 millones de dólares y su deuda externa aproximadamente 313.580 millones de dólares (Arena, 2011).

La evolución de la integración muestra la capacidad de forjar la identidad latinoamericana, uniendo esos colores de la diversidad y las asimetrías; aunque se han alcanzado distintos grados de integración, se ha conseguido el reconocimiento y el respeto del otro en la complementariedad y la diversidad, con ello ha logrado la fuerza para competir en un mundo globalizado.

No obstante lo anterior, es plausible el interés mostrado por los Estados, para que en cada uno de los organismos se logren acuerdos que permitan alcanzar el desarrollo en sus países y la coordinación entre todos los procesos, como se puede observar en cada caso.

\section{LA COMUNIDAD ANDINA (CAN)}

Mediante el Acuerdo de Cartagena o Pacto Andino, en 1962 se concreta este esfuerzo de Integración Subregional, con la participación inicial de Bolivia, Colombia, Perú, Ecuador y Chile. En 1973, Venezuela ingresa. $\mathrm{Su}$ finalidad es la liberación de comercio de bienes en la subregión, que les brinde autonomía y desarrollo.

En el 2003, mediante el Protocolo de Sucre, se modifica el Acuerdo de Cartagena; en el año 2004 que se firma el Acuerdo de Libre Comercio can-mercosur y para el 2006, se concreta una zona de libre comercio.

La can desde sus inicios ha tenido modificaciones $y$ adecuaciones en sus prioridades, siendo de especial preocupación, una integración comercial que permita alcanzar un máximo desarrollo, fortalecer la cooperación política y atender los problemas sociales. Puede afirmarse que la can ha sido el pilar fundamental en el establecimiento de otros sistemas de integración.

La decisión adoptada en el 2011 por los jefes de Estado del CAN, para iniciar una coordinación conjunta entre todas las Secretarías Generales; es decir, entre la CAN, MERCOSUR y UNASUR, que permita conocer elementos comunes y diferencias en cada uno de estos órganos; con miras a la articulación de una fuerza en común, es un paso importante. El reconocer que la fragmentación es uno de los elementos que juega en contra de la integración, es un gran avance, dado que no es lo mismo tener una sola institucionalidad, recursos humanos y mecanismos, que tener tres instancias donde intervienen en su mayoría los mismos actores. 


\section{MERCADO COMÚN DEL SUR (MERCOSUR)}

El Tratado de Asunción de marzo de 1991 da origen al Mercado Común del Sur (MERCosur), un órgano de carácter gubernamental con el propósito de formar una unión aduanera, restricciones arancelarias, política comercial común, además de temas industriales, fiscales, monetarios, transporte, comunicación y agrícolas. Está integrado por Argentina, Brasil, Uruguay, Paraguay y Venezuela, este último adquiere su condición de miembro de pleno derecho en 2012; sin embargo, en el 2016 es suspendido, en el caso de Bolivia es miembro pleno desde el 2015, Ecuador está en condición de asociado desde el 2004 $y$ en el 2011 solicita adquirir la condición de miembro pleno. En el caso de Guyana y Surinam logran la condición de Estados Asociados a partir del 2013.

En 1994, se firma el Protocolo de Ouro Pretox, que establece la base institucional de MERcosur y en el año 2002, se adopta el Protocolo de los Olivos para solucionar controversias, el cual fue reglamentado en el 2003. En el 2005, se crea el Fondo para la Convergencia Estructural del mercosur (Fосем), con la finalidad de financiar proyectos para promover competitividad, cohesión social y reducción de asimetrías entre los países miembros. En el 2005, se firma el protocolo constitutivo del PARLASUR.

Los objetivo primordiales del MERcosur son los aspectos comerciales, aduaneros $y$ arancelarios; su creación ha propiciado abrir nuevos espacios que beneficien a la población en otros temas. Un aspecto a destacar es la normativa jurídica como es el Protocolo de Brasilia de 1991, que permite la solución pacífica de las controversias $y$ es un referente interesante en esta materia (mercosur, 2016).

EL mercosur avanza en su propósito de ampliar las oportunidades paras sus miembros de forma competitiva, con múltiples acuerdos con países o grupos de países, con una estrategia de crear diversos organismos que le permitan implementar las políticas regionales y las iniciativas de los países.

México desde el año 2002, es un aliado importante para la región, al mantener una estrecha relación con los países del mercosur, firmando acuerdos de Complementación Económica, especialmente en materia de comercio, bienes y servicios.

\section{LA UNIÓN DE NACIONES SURAMERICANAS (UNASUR)}

unASUR es una propuesta de integración en los diversos temas de interés político, económico, cultural, social, ambiental, energético y de infraestructura, que permita forjar una identidad y ciudadanía suramericana.

Surge en la Cumbre de Isla Margarita (Venezuela) el 17 de abril de 2007 y se protocoliza en Brasilia en Mayo de 2008, conformado por doce países de América del Sur.

Como plantea Esparza (2008), la "integración suramericana nació como un gran proyecto de desarrollo e inserción internacional, como tal, resulta indispensable incorporar desde sus primeros pasos, el concepto de reducción de asimetrías como una condición propia de su naturaleza, de manera que los países puedan considerar este proyecto como legítimo" (Esparza, 2008).

El contexto político y económico de unASUR tiene la visión de mandatarios de izquierda, que buscan ambiciosos objetivos de la integración, con una agenda económica, política y social, que abarca entre otros temas: democracia, políticos, sociales, ambientales, culturales, comerciales, industriales, financieros, de cooperación internacional, consolidación de identidad, tecnología y participación ciudadana.

La visión estratégica contemplada en 21 objetivos específicos, le permite identificar los temas que interesan a la población y aquellos que se requieren para consolidar la integración.

unASUR logra articular además temas como la lucha contra los efectos del cambio climático, medidas de prevención de desastres; sin dejar de atender mecanismos que le permitan con eficiencia y eficacia superar las desigualdades sociales, de seguridad, migración, ampliar el acceso de la tecnología, la cooperación económica y comercial, la lucha contra el terrorismo y las drogas.

Estos objetivos expresan en lo formal, una voluntad política integracionista que 
promueve la cooperación entre sus miembros; sin embargo, los cambios de poder político, coloca a este espacio de integración en una fragilidad y se abre la posibilidad de volver la mirada a otros mecanismos de integración; no obstante, dependerá en gran medida del liderazgo de los nuevos mandatarios, $y$ su interés real para transformar las dificultades en oportunidades $y$ reforzar los esfuerzos colectivos en los procesos integracionistas.

LA ALIANZA BOLIVARIANA PARA LOS PUEBLOS DE NUESTRA AMÉRICA (ALBA)

Surge como iniciativa de la República Bolivariana de Venezuela, liderada por el Presidente Hugo Chávez, que retoma su sucesor el Presidente Nicolás Maduro para impulsar otra alternativa de integración para América Latina y el Caribe, frente a la propuesta del Área de Libre Comercio para las Américas (ALCA). Esta Alianza se sustenta en los principios ideológicos de unidad latinoamericana, el respeto a la soberanía de los pueblos, la solidaridad y en la complementariedad de las economías de los países que la integran.

El alba entra en el escenario como la posibilidad de alcanzar una estrategia común, para adoptar un modelo de integración que incluya temas económicos, sociales y culturales; entre estos, el desarrollo sostenible de los recursos, la seguridad y la soberanía alimentaria, laenergía, temas como la cultura, la salud, la educación y la cooperación técnica.

Sin embargo, este gobierno de Nicolás Maduro enfrenta serios cuestionamientos de violación a los derechos humanos, carencia de alimentos, medicinas y persecución a líderes de oposición política, que ha llevado a la directiva del Parlamento Venezolano a pedir la intervención de la oes para aplicar la Carta Democrática por alteración del orden constitucional y ventilar el caso de Venezuela ante el Consejo Permanente.

Con la crisis política, social y económica de República Bolivariana de Venezuela, no se vislumbra en plazo inmediato que pueda evolucionar hacia su fortalecimiento; sin embargo, es prematuro concluir sobre el futuro del ALBA.

\section{4) EVOLUCIÓN DE LA INTEGRACIÓN EN EL} CARIBE

\section{LA COMUNIDAD DEL CARIBE (CARICOM)}

CARICOM (por sus siglas en inglés), fundada en 1973, sustituye la Asociación de Libre Comercio del Caribe, siendo sus antecesor la Federación de las Indias Orientales. Mediante la firma el Tratado de Chaguaramas, se estableció la Comunidad del Caribe y el Mercado Común. Dicho tratado entró en vigor el día primero de agosto de 1973 con las ratificaciones de Barbados, Jamaica, Guyana y Trinidad y Tobago.

Tiene 15 miembros plenos y cinco miembros asociados. Lo integran países, en su mayoría excolonias inglesas: Antigua, Bahamas, Barbados, Belice, Dominica, Granada, Guyana, Jamaica, Montserrat (colonia británica), San Cristóbal y Nieves, San Vicente y las Granadinas, Santa Lucía, Surinam (antigua Guyana Holandesa), Trinidad y Tobago y Haití (Red, 2016).

Dentro de su estructura institucional, la Conferencia de Jefes de Gobierno es su órgano superior, a quien le compete establecer la política de la comunidad.

CARICOM ha logrado establecer distintas instituciones que atienden temas diversos, como desastres y emergencias, alimentación, medio ambiente, agricultura, desarrollo, entre otras. Comparte con el can, algunos de los objetivos integracionistas como el fortalecimiento de su comercio exterior, las relaciones del comercio y el mercado.

Cuba no forma parte del CARICOM; sin embargo, mantienen negociaciones en base al Acuerdo Comercial y de Cooperación Económica, y se avanza en acuerdos para la promoción y protección de inversiones, como lo evidenciado en la v y vi Cumbre савIсом-Cuba. Fortalecer estas relaciones es un proceso importante, por los vínculos geográficos e históricos de estas naciones Caribeñas.

Las relaciones exteriores de este país no han sido fáciles, con toda la complejidad política y económica que debió padecer el pueblo cubano para resistir $y$ defender su soberanía como resultado del bloqueo sufrido después de 
la Revolución por parte de Estados Unidos y su poder de incidencia en la región para impedir estos esfuerzos integracionistas.

Son muchos aportes de los países caribeños al modelo que se ha ido creando en la región CARICOM. Sin duda que enfrentar grandes mercados requiere de un balance entre beneficios conjunto y manejos de soberanías en los países, entendiendo que sus pequeños mercados deben abrirse al mundo, pero tienen en su contra que los precios de sus productos responden a otras dinámicas del mercado internacional, en condiciones desventajosas: "En el caso de las pequeñas economías del Caribe, esta vulnerabilidad se acrecienta al sumarse la presencias de fenómenos meteorológicos tales como ciclones que en varias ocasiones han devastado la infraestructura económica de estos países" (Reyes, 2007).

No importa a que región se pertenece, la geografía está diferenciada por líneas imaginarias que no están por encima de la capacidad y sueños de trabajar unidos por garantizar el bienestar de los pueblos y lograr una sola América.

Los tiempos que se viven, deben ser los tiempos de la igualdad $y$ de equidad entre los género, para ello, se requiere de una integración latinoamericana y caribeña, capaz de lograr una agenda a partir de estos derechos. La integración debe servir a través de sus distintos órganos para generar mecanismos eficientes de protección de los distintos ecosistemas mediante códigos éticos de explotación, unificar criterios sobre la protección, uso y sostenibilidad del medio ambiente; cuidar el agua, la producción de energía limpia y evitar la minería al cielo abierto.

Los efectos de la globalización y el libre comercio, el cambio climático y la crisis alimentaria, demanda asegurar la propiedad de los recursos naturales, especialmente de los países desarrollados, aunado a buscar solución a complejos problemas que afectan a la población: el transporte y la comunicación; los servicio sociales como salud, educación, vivienda, seguridad ciudadana; el fortalecimiento de la identidad y la cultura; la confianza y solidaridad para buscar estrategias $y$ acciones comunes para el buen vivir, es decir, una agenda concertada a largo plazo como tema de Estado; esa es la arquitectura de integración que se busca.

RETOS Y PERSPECTIVAS DE LOS PROCESOS DE INTEGRACIÓN EN AMÉRICA LATINA Y EL CARIBE

RETOS

La integración requiere de real voluntad política de los gobiernos de América Latina $y$ el Caribe para acordar una agenda integral que abarque los temas del desarrollo humano sostenible con enfoque de derechos humanos: la economía, el comercio, problemas sociales, ambientales, energéticos, de comunicaciones, seguridad, migratorios, la institucionalidad, su estructura, mecanismos de coordinación, recursos para su funcionamiento, entre otros.

Hace falta mirar hacia adentro de los Estados y responder a las necesidades de la población; hacer un alto para buscar una estrategia que permita sacar ventaja de la diversidad del sistema; aunque con distintos procesos, todos tienen a su haber la concreción de acuerdos, marcos normativos, estructuras de coordinación gubernamental, convenios con Estados y organismos de otras regiones del mundo. Mientras se mantenga la fragmentación y la multiplicidad de órganos de integración, muchos de los cuales tienen a los mismos actores, será difícil tener una agenda común.

Otro reto de gran prioridad para los pueblos, es la desigualdad social, de género, clase, condición y territorio, esto pasa por compartir un marco normativo común en materia de derechos humanos; sin dejar de mencionar el tema de corrupción a nivel nacional e internacional. Sin duda, se podría hacer distintos cuestionamientos a la integración en la región, pero en la evolución de cada uno, hay que reconocer que todos tienen un acumulado de experiencias que aportan luces en un nuevo modelo de integración.

Hay que fortalecer la presencia favorable de México y otros países de la Unión Europea como observadores o miembros de los espacio de integración. Las asimetrías entre los Estados y a lo interno de sus territorios, es un desafio para la integración; es una tarea titánica que requiere de liderazgo político y trabajo permanente. 
Se tiene que ser capaz de atender los temas de conflicto, centrado en los factores que unen, desde la solidaridad y hermandad de los pueblos, con agendas de concertación propias, con mecanismos eficientes y eficaces para la resolución pacífica de conflictos y consolidar las instancias diplomáticas para evitar que se profundicen reclamaciones entre los Estados latinoamericanos y caribeños, superando disputas como las de Bolivia con Perú y Chile; Costa Rica y Nicaragua, y este último con Colombia.

Fortalecer los Estados Nacionales con participación ciudadana, requiere lograr una estructura que facilite la representatividad de todos los actores sociales, incluyendo la sociedad civil y movimientos sociales, que aporten sus visiones, debatan sobre la integración, tengan el respaldo y legitimidad para avanzar como expresión de un ejercicio democratizador y no como un espacio político ajeno a la población.

Los sistemas de integración tienen el reto de propiciar agendas y adoptar políticas públicas regionales que impacten las políticas nacionales en temas sociales como: pobreza, salud, educación, vivienda, agua, respeto a los pueblos indígenas, afrodescendientes y minorías étnicas, los migrantes, refugiados, víctimas de violencia en todas sus formas y la especial atención a problemas estructurales como la desigualdad y la exclusión social.

Con un clima de respeto a las diferencias, se está en la capacidad de hacer un alto y analizar todos los órganos, procurando evitar la duplicidad de esfuerzos y la burocracia; esto sería el inicio de otra etapa en la integración, para construir desde la unidad una agenda transformadora capaz de mostrar la unidad de la región.

Otro de los principales retos de los procesos de integración de América Latina y el Caribe, es superar la crisis política y de gobernanza que existe en la mayoría de los países, que lesiona la credibilidad de la población de los verdaderas intenciones de sus principales líderes, especialmente en Centro y Suramérica, donde los casos de corrupción han llevado al juzgamiento de Jefes de Estado, Ministros, Magistrados $y$ funcionarios de distintos niveles que han utilizado el poder para su enriquecimiento con recursos del Estado.

Los escándalos de corrupción en los países de la región afectan la integración, no solo porque se somete a cuestionamientos la necesidad de mantener estos organismos; sino porque se ven como espacios para hacer relaciones diplomáticas y concretar intereses que nada tienen que ver con resolver los problemas de la población, agravando la fragilidad que arrastran.

La corrupción además de atentar contra los intereses de las grandes mayorías, de forma indirecta y directa debilita la credibilidad de los sistemas de integración, en la medida que se vuelven un espacio que genera algún tipo de obstáculos en los procesos locales de persecución del delito. Tal es el caso emblemático de Panamá, cuyo expresidente Ricardo Martinelli tiene varios procesos, pero se ha tratado de amparar en la inmunidad que le otorga el ser diputado del Parlamento Centroamericano, hecho público y notorio.

De igual forma, la salida por casos de corrupción del presidente $y$ vicepresidenta de Guatemala, y el juzgamiento del Expresidente Flores de El Salvador, que falleció antes de ser procesado, por lo cual no se puede concluir sobre su responsabilidad penal de los cargos imputados. Todos estos mandatarios tuvieron un papel importante en el fortalecimiento del sistema de integración centroamericana. También se observa como un reto, el actual escenario político con la llegada de nuevos mandatarios, por lo cual se espera ver como se consolidan estos espacios.

La situación política en los países suramericanos pone a prueba las democracias. El caso de las presidentas, Dilma Russeff de Brasil $y$ Michel Bachelet de Chile, ambas con un fuerte rechazo popular; $y$ en el caso de Brasil, que ha sido destituida de su cargo como mandataria por las decisiones en el uso de fondos públicos y la adopción de otras medidas; sin duda, son situaciones que inciden el quehacer de los sistemas de integración en el Sur; quedan pendiente concretar temas importantes, ya que se trata de países con las economías más fuertes que lideran el MERCOSUR, UNASUR y el ALCA. 
Aunado a lo anterior, el término del periodo presidencial de mandatarios de ideas socialistas como José "Pepe" Mujica de Uruguay, limitan la propuesta de transformación de la integración; su liderazgo aportaba ideas progresistas a una agenda social y era la voz permanente de alcanzar la unidad desde la visión Bolivariana.

En el caso de la expresidenta Argentina, Cristina Fernández de Kisner, hoy cuestionada por el actual presidente Mauricio Macri, se mantuvo como una aliada importante de las iniciativas de la República Bolivariana de Venezuela, en el alBa y unasur. Contrario a esa posición, su sucesor expresa su intención de insistir en los reclamos para que el gobierno de Nicolás Maduro libere los presos políticos y apuesta por fortalecer los lazos comerciales con la Alianza Pacífico y la Unión Europea (NoDAL, 2015).

Lo anterior no significa que los nuevos mandatarios de la región Sur, no estén dispuestos a retomar las propuestas sobre temas sociales, ambientales, de derechos humanos; sino el tener claro que históricamente han impulsado como prioridad los temas económicos, ligados al comercio, mercado, aduaneros, migratorios, energía, entre otros.

\section{PERSPECTIVAS}

Se debe valorar el trabajo integracionista con sus aciertos y desaciertos; hay buenas prácticas $y$ lecciones aprendidas que mostrar, aunque hay un largo camino para alcanzar normativas, instrumentos y mecanismos que permitan la complementariedad y el fortalecimiento de las instancias económicas, comerciales, sociales, culturales, ambientales y del mercado, que fueron iniciados.

Los Estados de América Latina y el Caribe tienen en sus manos decidir el futuro del espacio común de integración de toda la región $y$ consolidar los esfuerzos. El continuar destinando recursos humanos y económicos para mantener órganos con funciones similares son ineficientes $y$ burocráticos. Los sistemas de integración de nuestra región, conservan un poder político; sin embargo, cada iniciativa de los subsistemas, donde coinciden la mayoría de los países miembros, aleja la posibilidad de concretar esa visión y agenda común que den respuestas a los problemas más persistentes de la población.

El proceso de integración debe buscar el fortalecimiento de la democracia y el respeto a la soberanía de los pueblos; es un imperativo político y ético trascender prácticas de favorecer intereses foráneos para trabajar por las sociedades en la solución de los problemas sociales, culturales y ambientales, procurando políticas públicas que aseguren un desarrollo humano sostenible.

El futuro de una América unificada como soñaron los libertadores, ha sido parte del proceso de integración. Las distintas expresiones de los órganos creados en las subregiones, dan cuenta en su evolución histórica que la región ha persistido en crear alianzas $y$ acuerdos para enfrentar los embates ideológicos, económicos, políticos y de la globalización, desde sus propias realidades.

La integración requiere de líderes con capacidad de ver estos espacios como oportunidades de desarrollo para los pueblos, la defensa de sus culturas y recursos; con objetivos comunes, entendiendo que son procesos complejos y requieren de una vocación de trabajar en alianzas, con respeto y solidaridad. La dinámica de las sociedades requiere crear estrategias novedosas, dejando de lado las especificidades para reconocer sus fortalezas y debilidades.

Los problemas comunes deben guiar a los tomadores de decisiones, de manera que la integración se convierta en el mecanismo idóneo para buscar respuestas concertadas, solidarias y equitativas, superando las burocracias y los tecnicismos, que en muchos casos son impedimentos en la búsqueda de una identidad entre los países que garanticen el bienestar y los derechos humanos.

El futuro de los pueblos aunado a la integración, debe llevar a fortalecer alianzas con otros Estados, como México y Canadá, los cuales apoyan el desarrollo comercial, económico y social de la región. Asimismo, con Cuba, que tras el acuerdo con Estados Unidos de Norteamérica para el restablecimiento de las relaciones, es un importante actor en los temas sociales. De igual forma, se debe seguir consolidando las relaciones con otros países 
iberoamericanos, de la Unión Europea y Asia, donde algunos ya participan en calidad de observadores.

\section{CONCLUSIONES}

El proceso de integración latinoamericana $y$ caribeña ha tomado décadas; ha sido un largo proceso cuya evolución tiene avances $y$ retrocesos, sin que se pueda aún concretar el sueño de lograr la unidad de América. La integración ha tenido que superar situaciones difíciles, como la desigualdad, la pobreza, el cambio climático, la seguridad alimentaria, la justicia, la democracia, la participación ciudadana, la corrupción, entre otras; pero sus logros se pueden constatar en la constitución de órganos de coordinación, mecanismos, normas jurídicas, instancias para la solución de los conflictos y alianzas, con la finalidad de alcanzar consensos en temas económicos, comerciales, de mercado, aduaneros, arancelarios, migratorios, ambientales, culturales y más recientemente abarcando distintos problemas comunes.

Los procesos de integración muestran una evolución compleja, pero hay que encontrar el camino común para el futuro, producir los cambios en los órganos, mecanismos y normativas, que no representen duplicidad de esfuerzos; las múltiples instancias generan un sinnúmero de acuerdos y dificulta la sostenibilidad. Se pretende una integración que genere oportunidades de desarrollo para los pueblos, el bienestar social, la paz, la justicia y la democracia.

\section{REFERENCIAS}

acan efe/la prensa (26 de junio de 2015.). Cancilleres sica-resaltan importancia de fortalecer la economía de la región. La Prensa. Recuperado de http://www.prensa.com/economia/ Cancilleres-SICA-resaltan-importanciafortalecer_0_4240826039.html

Agencia Efe (17 de julio de 2015). Alianza del Pacífico falta a la cita del mercosur. Agencia EFE. Recuperado de http:// www.efe.com/efe/america/economia/ la-alianza-del-pacifico-falta-a-citamercosur/20000011-2667364
Alianza del Pacífico (07 de junio de 2016). Negocios e Inversión. Recuperado de http://www.alianzadelpacifico.net

Andina, C. (13 de diciembre de 2016). COMUNIDAD ANDINA. Recuperado de http// comunidadandina.org

Arena, S. (03 de abril de 2011). La Integración en América Latina: Una Lucha desde los siglos xvIII $y$ xix hasta nuestros días. Recuperado de http://wwwserenas29. blogspot.com

Asociación Latinoamericana de Integración (ALADI). (2 de julio de 2015). ¿Qué es la ALAdI? Recuperado de http://www.aladi. org/nsfaladi/preguntasfrecuentes.nsf/7 fc5dc8b0352c1032567bb004f8e78/fe13 9 cfd067aec28032574be0043f17e?Open Document

Bolívar, S. (2015). Carta de Jamaica. Caracas: Comisión Presidencial para la Conmemoración del Bicentenario de la Carta de Jamaica.

Castro, F. (5 de mayo de 1959). Discurso del Comandante Fidel Castro Ruiz en Montevideo. Recuperado de http://www. cuba.cu/gobierno/discursos/1959/esp/ f050559e.html

Castro, F. (17 de agosto de 2006). Cubal Gobierno/Discursos. Recuperado de http://www.cuba.cu/gobierno/ discursos/1959/esp/f050559e.html

Celac (17 de Diciembre de 2016). CELAC-Comunidad de Estados Latinoamericanos y Caribeños Recuperado de http//www.sela.org

Comisión Económica para América Latina $y$ el Caribe (CEPAL) (12 de octubre de 1999). La Conformación del Area de Libre Comercio. (ALCA) Aspectos Institucionales y comerciales. Recuperado de http://repositorio.cepal. org/bitstream/handle/11362/2729/1/ LCmexL405_es.pdf

cepal (24 de noviembre de 2014). Comisión Económica para América Latina y el Caribe. Recuperado de https://www. cepal.org/es/comunicados/alianza-delpacifico-y-mercosur-representan-masde-80-del-comercio-exterior-regional 
соммса (18 de Diciembre de 2016). соммса Consejo de Ministras de la Mujer de Centroamerica y Republica Dominicana. Recuperado de http//www.sica.int/ commca

De la Mora, L. M. y Rodríguez, D. (2011). Por qué vale la pena repensar la integración latinoamericana. Integracion \& Comercio (33), 1-15.

Esparza, M. E. (2008). Las asimetrías y el proceso de integración suramericana. Revista de la integración (2), 82-92.

EFE, A. (17 de julio de 2015). Alianza del Pacifico Falta a la Cita del Mercosur. Recuperado de http://www.msn.com/et/ noticias

Fabra, U. d. (17 de mayo de 2012). Los Procesos de integracion de América Latina. CARICOM. Recuperado de http;// wwwufp,educ/integracionamerica/ caricom

Fernández Nadal, E. (2014). Independencia y unión: dos aspectos de la utopía emancipatoria hispanoamericana a lo largo del siglo xix. En G. Hoyos y S. Villavicencio. La Unión Latinoamericanana: diversidad y politica (pp.233-255). Buenos Aires, Argentina: CLACSO.

Fernández Rosas, J. C. (2000). Los Modelos de Integración en América Latina y el Caribe y el Derecho Internacional. Madrid: во上.

García, J. M. (1979). Augusto Cesar Sandino: realización del sueño de Bolivar. México: Porrúa.

Granma, R. I. (28 de enero de 2015). Celac: una Voz Latinoamericana y Caribeña. Granma. Recuperado de http://www. granma.cu/mundo/2015-01-28/celacuna-sola-voz-latinoamericana-y-caribena Juventud, P. (5 de mayo de 2009). Carta de Jamaica Simón Bolívar. Recuperado de httpp://wwwjuventud.psup.org.ve

mercosur (18 de diciembre de 2016). En Pocas

Palabras ¿Qué es el MERCOSUR? Recuperado de http//www.mercosur.int

NODAL (21 de diciembre de 2015). Mercosur. Recuperado de http://www.nodal. am/2015/12/sin-la-presencia-de-maduro$y$-con-el-debut-internacional-de-macricomienza-una-nueva-cumbre-delmercosur/

odeca (13 de diciembre de 1991). Sistema de Información sobre Comercio Exterior. Recuperado de http://www.sice.oas.org/ trade/sica/SG121391.asp

Parlamento Centroamericano (parlacen) (7 de julio de 2015). Atribuciones y Competencias. Parlamento Centromericano. Recuperado de http://www.parlacen. int/Informaci\% C 3\% B 3nGeneral/ AtribucionesyCompetencias.aspx

Piera, L. (1 de agosto de 1991). En pocas palabras ¿Qué es el MERCosur?. Recuperado de http://www.mercosur. int/innovaportal/v/3862/2/innova.front/ en-pocas-palabras

Pizarro, J. (2 de agosto de 1991). Estatuto del Parlamento Latinoamericano. Recuperado de http://www.parlatino.org/ pdf/documentos/estatuto-parlatino.pdf

Primerísima, R. 1. (20 de diciembre de 2007). La Gente Radio la Primerísima. Recuperado de http//wwwradiolaprimerisima.com/ noticias/

Red, E. (19 de diciembre de 2016). CARICOM. Recuperado de http://www.ecured.cu

Reyes, G. E. (2007). Procesos de Integración de América Latina y el Caribe: caracterización general y potencialidad de nuevos ejes de integración. Tendencias, VIII (2), pp.7-42.

Rubio, M. (2011). Globalización y su impacto sobre las estrategias de gestión de las empresas cooperativas. Estudio de casos en el ámbito Chileno (Tesis Doctoral en economía aplicada). Valencia: Universidad de Valencia.

Secretaría de Integración Económica Centroamericana (SIECA). (21 de marzo de 2001). Secretaría de Integración Ecconómica. Recuperado de http://www. sieca.int

sica (20 de julio de 2010). sica Cumbres. Recuperado de http://www.sica.int/ busqueda/Reuniones $\% 20$ Grupo $\% 20$ de $\% 20$ Autoridades.aspx? IDItem $=83175$ 
$\& I D C a t=9 \& I d E n t=401 \& I d m=1 \& I d m S t$ yle $=1$

Universitat Pompeu Fabra (17 de mayo de 2012). Los Procesos de integración de América Latina. СARICOM. Recuperado de http;//wwwufp,educ/integracionamerica/ caricom

Vacchino, J. M. (17 de diciembre de 1990). La dimensión parlamentaria de los procesos de integración regional. Tomo II. Buenos Aires: BID-INTAL.
Wikipedia (3 de julio de 2015). Comunidad de Estados Latinoamericanos y Caribeños. Recuperado de https:// es.wikipedia.org/wiki/Comunidad_ de_Estados_Latinoamericanos_y_ Caribe\%C3\%B1os

Fecha de ingreso: $30 / 11 / 2016$ Fecha de aprobación: 19/05/2017 
